\title{
Prevalencia de Infestación por Toxoplasma Gondii en un Grupo de Madres y sus Productos de Gestación
}

\author{
Drs.: Clemencia Fernández de Guardiola y María Cristina Cerón Cerón \\ Hospital Infantil Lorencita Villegas de Santos \\ Bogotá, Colombia 1981
}

\section{Introducción}

El parásito toxoplasma Gondii fue descubierto hace aproximadamente 70 años en roedores de Africa y América; posteriormente, se discutieron otros hallazgos en Europa en especies de mamíferos no roedores $(1-2)$.

La primera referencia de esta parasitosis en humanos se informó 11 años después y en ella se describió la existencia de quistes de Toxoplasma Gondii en la retina de un lactante; 16 años después, en 1939, se comprobó la trasmisibilidad del parásito, hombre animal, mediante inyección de un lisado de tejidos obtenidos de un neonato, cuya muerte fue causada por encefalitis toxoplasmósica, a un animal de experimentación; esto sugirió, dada la edad del lactante (neonato), la posibilidad de adquisición de la enfermedad durante la vida extrauterina (2).

La patogenia de la enfermedad fue descrita en 1941, en términos de una triada compuesta por coroidoretinitis, microcefalia y calcificaciones cerebrales con signos clínicos de daño cerebral. Posteriormente, la adquisición de técnicas cada vez más específicas y depuradas de diagnóstico de laboratorio, ha demostrado una prevalencia de amplia variabilidad que va desde el $1 \%$ en esquimales, hasta el $95 \%$ en Tahitianos. Los valores de prevalencia intermedios descritos van desde el $4 \%$ en indios navajos hasta el $85 \%$ en la población adulta de parisienses, mientras en la población sueca se informan valores del $50 \% \quad(2-3)$.

Es llamativo el hecho de que en los Estados Unidos, del total de 66 millones de personas afectadas de parasitosis por Toxoplasma, el mayor porcentaje de casos se encuentra después de los 10 años de la vida; mientras el menor se informa en las edades anteriores a ésta. Lo anterior, si bien representa variaciones en la susceptibilidad y en el riesgo de adquirir la enfermedad, más importantes en la medida en que la edad progresa, supone a su vez condiciones ecológicas diferentes para el adulto en 
relación al niño, desde el punto de vista de infestación y desarrollo de la enfermedad; sin embargo, la posibilidad de adquirir la enfermedad en la vida intrauterina hace necesario investigar la prevalencia de esta enfermedad en adultos, considerando a éstos como factores directos de transmisión humana (2).

En Colombia los estudios desarrollados, si bien su representatividad no es absolutamente válida en términos tanto de muestra como de relaciones ambientales y agentales-hostales, demuestran la existencia del problema. El hecho conocido de la irreversibilidad de las secuelas de la enfermedad, tanto como la posibilidad de la existencia de reservorios diferentes a los descritos, amerita realizar investigaciones, inicialmente de tipo descriptivo, con el fin de establecer la magnitud de este problema en el país con fines de, posteriormente, llevar a cabo estudios anal íticos $\mathrm{y} / \mathrm{o}$ experimentales dentro de un criterio eminentemente eugenésico y de tipo preventivo (2 a 10).

\section{ii. Objetivos}

1. Establecer la prevalencia de toxoplasmosis en la población de mujeres adultas que asisten a la consulta de Ginecología y Obstetricia del Hospital Infantil Lorencita Villegas de Santos de Bogotá, Colombia, durante la gestación.

2. Establecer la frecuencia de infección intrauterina por toxoplasma en losproductos de madres infestadas.

3. Relacionar las secuelas causadas por el parásito en los productos de gestación, con el trimestre de embarazo en que se produjo la infestación.

4. Establecer la frecuencia de infestación materna con la enfermedad activa.

\footnotetext{
* Este trabajo fue posible gracias a la colaboración de Laboratorios Specia de Colombia.
}

5. Conocer las características inmunológicas e inmunitarias del recién nacido hijo de madre infestada.

6. Establecer las relaciones existentes entre los títulos de antitoxoplasma en la madre $y$ et niño con secuelas clínicas.

\section{Material y Métodos}

\section{A. MATERIAL:}

Estuvo constituido por 500 madres gestantes y sus productos de gestación, del total de 10.000 asistentes a la consulta de Ginecología y Obstetricia del Hospital Infantil Lorencita Villegas de Santos de Bogotá, Colombia, durante el lapso comprendido entre el 10. de febrero y el 1o. de agosto de 1981.

De las asistentes se seleccionó arbitrariamente una de cada veinte madres, previa explicación del programa y su aceptación. Con fines de referencia, desde el punto de vista inmunológico, se estudiaron dos neonatos del Servicio de Recién Nacidos del Hospital Infantil Lorencita Villegas de Santos, en los cuales se sospechó la infestación.

\section{B. METODOS:}

En cada una de las madres se explicó el siguiente procedimiento:

\section{Clasificación del estado socio-econó- mico según el siguiente criterio:}

Clase A: Sin ingreso fijo, ni profesión, ni vivienda propia, más de 3 personas a cargo.

En las madres cuyos títulos fueron iguales a 1:256 no se instauró tratamiento alguno y se tomó un nuevo control serológico al mes; en el caso de encontrarlo en ascenso se daría tratamiento como antes se explicó. 
En los productos de gestación de madres con títulos superiores a 1:256 se aplicó el siguiente procedimiento:

a) Determinación de títulos de anticuerpos antitoxoplasma específicos para $\lg \mathrm{G}$ e $\operatorname{Ig} \mathrm{M}$.

b) De acuerdo a los resultados informados, los niños que presentaron títulos de IgG superiores a 1:256 y/o IgM positivos en cualquier cifra, recibieron tratamiento consistente en Pirimetamina dosis de $1 \mathrm{mg}$ por kilogramo durante 15 días desde el nacimiento y Sulfadiazina en dosis de 150-200 mg por kilogramo durante 15 días, iniciando a los 15 días de nacido.

Posterior al tratamiento se realizó nuevo control de títulos de anticuerpos antitoxoplasma para observar las variaciones en términos de estabilización, ascenso o descenso, así: con títulos estabilizados o en descenso se consideraría el tratamiento como efectivo, continuando con controles mensuales hasta su descenso a cifras de 1:256 o inferiores. Títulos en ascenso: se consideraría el tratamiento como inefectivo, siendo necesario repetirlo. A los pacientes que recibieron la terapéutica arriba citada se les controló mediante la determinación de hemoglobina semanalmente, para detectar efectos colaterales de estas tales como mielodepresión; en el caso de encontrar cifras de hemoglobina de $8 \mathrm{mg} \mathrm{o}$ menores se les aplicaría a los pacientes una transfusión de glóbulos rojos empaquetados, a dosis de $10 \mathrm{cc} / \mathrm{kg}$.

c) En todos los niños se buscó la presencia de malformaciones congénitas, detectables al examen clínico y de lesiones encefálicas determinables por radiología, así como de coroidoretinitis por examen de fondo de ojo.

d) En todos los niños se determinaron los siguientes parámetros: peso en gramos, perímetro cefálico en centímetros, edad gestacional, signos de sufrimientos fetal, apgar al nacimiento y aparición de ictericia; en cuanto a la placenta, peso y caracter ísticas (37).

\section{Resultados}

El $43 \%$ de las madres estudiadas se clasificaron en la categoría B, $22.4 \%$ en la $A$, el $15.6 \%$ en la $C$ y una relación de $19.0 \%$ correspondió a la clasificación de semipensionado (Gráfica No. 1).

Gráfica No. 1

\section{GESTANTES ESTUDIADAS SEGUN CLASIFICACION SOCIOECONOMICA}

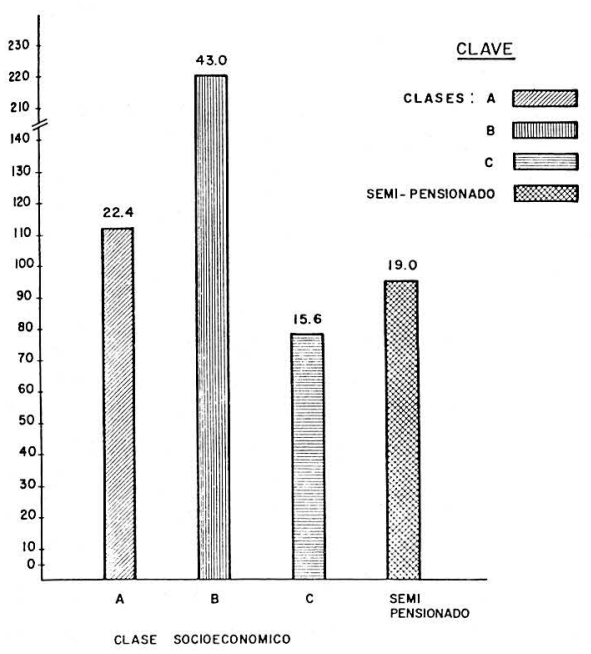

En las madres estudiadas el $57 \%$ presentó títulos no reactivos. El total de titulaciones reactivas se distribuyó en la forma siguiente: Títulos menores a $1: 256-38.4 \%$, títulos iguales a 1:256 $-3.2 \%$, títulos superiores a 1:256$1.4 \%$.

El total de títulos positivos fue de $43.0 \%$, (Gráfica No. 2). 
Distribución según edad gestacional y título de anticuerpos antitoxoplasma.

En cada trimestre de gestación la mayoría de madres presentaron títulos, no reactivos distribuidos así: primer trimestre 60 madres, segundo 85 y tercero 140

Los títulos reactivos se distribuyeron en la forma siguiente: inferiores a 1:256: primer trimestre 17 madres, segundo 75 y tercero 100. Con títulos superiores a 1:256 la distribución fue la siguiente: primer trimestre 0 madres, segundo 3 , tercero 4.

Con títulos iguales a 1:256 la distribución fue: primer trimestre 4 casos, segundo 8 y tercero 4. (Gráfica No. 3).

La relación de la historia de abortos de 2 o menos fue superior en las madres reactivas que en las no reactivas $(54.5 \%$ y $25.0 \%$ ). En las madres que tenían 3

\section{Gráfica No. 2}

\section{TITULOS ANTITOXOPLASMA EN 500 GESTANTES ESTUDIADAS}

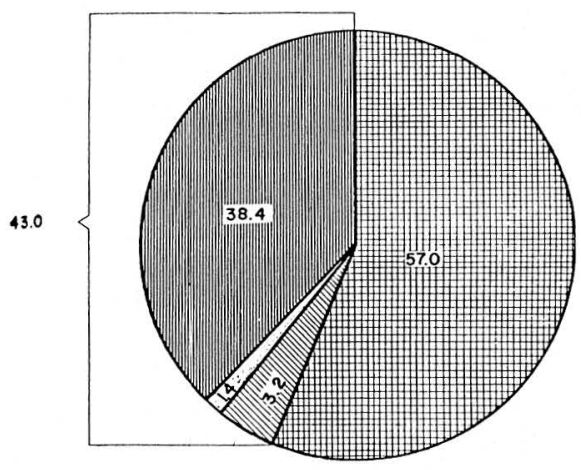

CLAVE TITULOS

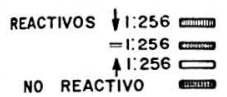

Gráfico No. 3

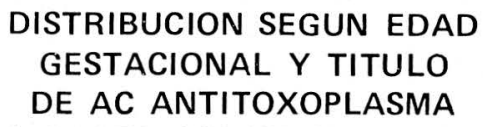

(BOGOTA COLOMBIA - 1981

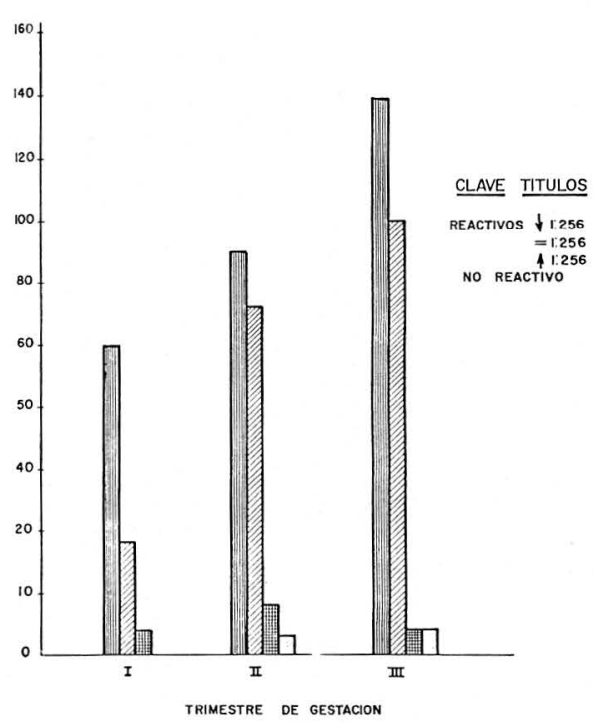

o más abortos la relación fue de $15.9 \%$ en las reactivas, contra $4.9 \%$ en las no reactivas. La relación del total de abortos fue de $70.4 \%$ en las madres con títulos reactivos y de $29.5 \%$ en las que ten ían títulos no reactivos, (Cuadro No. 1).

Cuadro No. 1

\section{RELACION DE ABORTOS CON LOS TITULOS ANTI-TOXOPLASMA}

\begin{tabular}{|c|c|c|c|c|c|c|}
\hline \multicolumn{7}{|c|}{ MADRES } \\
\hline \multicolumn{3}{|c|}{ REACTIVAS } & \multirow{2}{*}{$\frac{\text { NO }}{\text { Ne }}$} & \multirow{2}{*}{$\frac{\text { REACTIVAS }}{\%}$} & \multicolumn{2}{|r|}{ TOTAL } \\
\hline & № & $\%$ & & & № & $\%$ \\
\hline $\begin{array}{c}\text { ABORTO } \\
(\leq-2)\end{array}$ & 48 & 54. 5 & 22 & 25.0 & 70 & 79.5 \\
\hline $\begin{array}{c}\text { A BORTO } \\
(\geqq 3)\end{array}$ & 14 & 15.9 & 4 & 4.5 & 18 & 20.5 \\
\hline TOTAL & 62 & 70.4 & 26 & 29.5 & 88 & 100.0 \\
\hline
\end{tabular}


Del grupo de madres no reactivas, en el $44.2 \%$ no se encontró ninguna signología, en el $5.8 \%$ se encontró hipertemia, en el $3.4 \%$ rinorrea, en el $2.6 \%$ rash y el $1.0 \%$ presentó adenopatías.
En el grupo de madres reactivas el $20.8 \%$ no presentó signología, el $7.6 \%$ presentó hipertemia, el $7.0 \%$ rinorrea, el $5.2 \%$ rash y el $2.4 \%$ adenopatías, (Gráfica No. 4).

Gráfico No. 4

\section{FRECUENCIA Y RELACION PORCENTUAL DE SIGNOS CON \\ TITULOS ANTITOXOPLASMA (MADRES) \\ (BOGOTA - COLOMBIA 1981)}

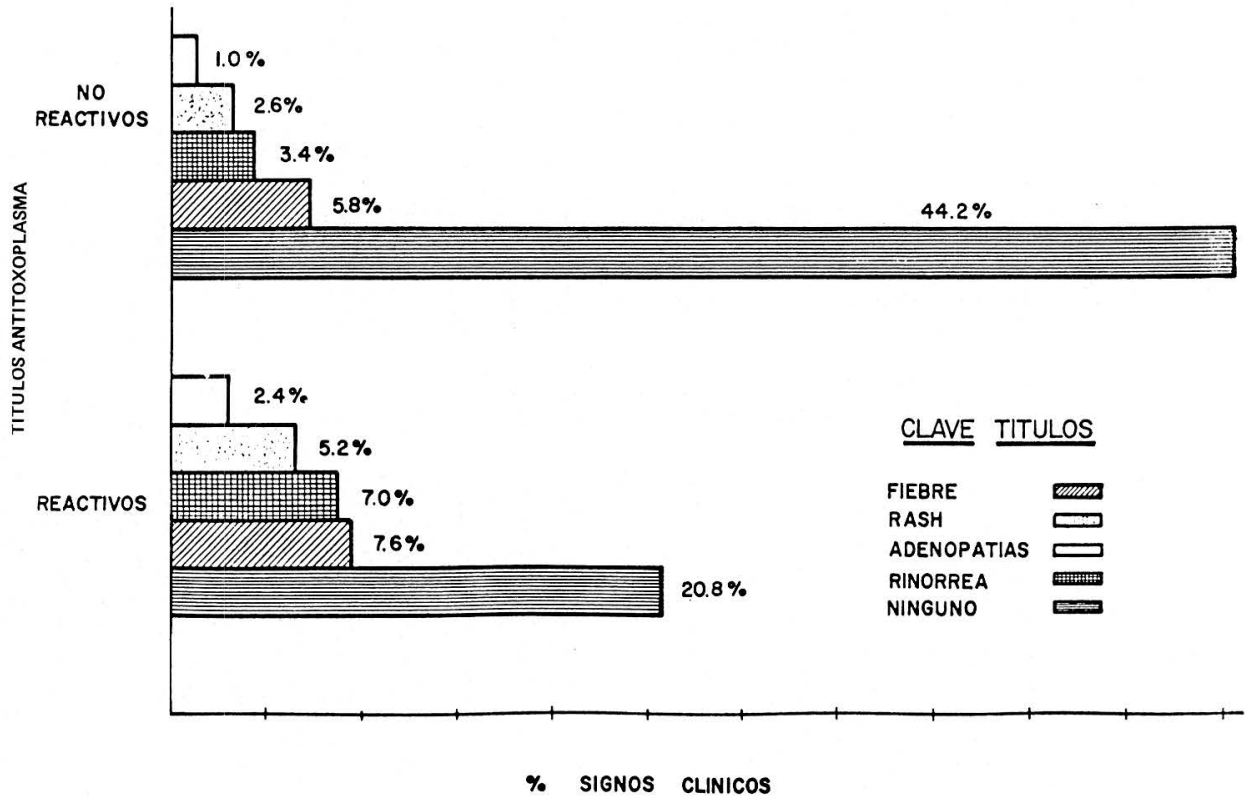

En 16 madres con títulos de 1:256, que no recibieron tratamiento, éste descendió espontáneamente en 11, de los cuales en 3 el descenso fue prácticamente de la mitad o menos, mientras en 8 éste fue aproximadamente de una tercera parte de los valores iniciales. En 4 madres el título se mantuvo sin variación y en 1 aumentó.
Siete madres con títulos positivos por encima de 1:256 recibieron tratamiento para la enfermedad; 3 de éstos descendieron por debajo del $50 \%$, mientras el descenso de las 4 restantes fue aproximadamente del $30 \%$, situándose a nivel de los valores considerados normales de 1:256; los demás descendieron por debajo de esta cifra, (Gráfica No. 6). 
Gráfico No. 5

\section{ANTICUERPOS ANTITOXOPLASMA EN MADRES QUE NO RECIBIERON TTO}

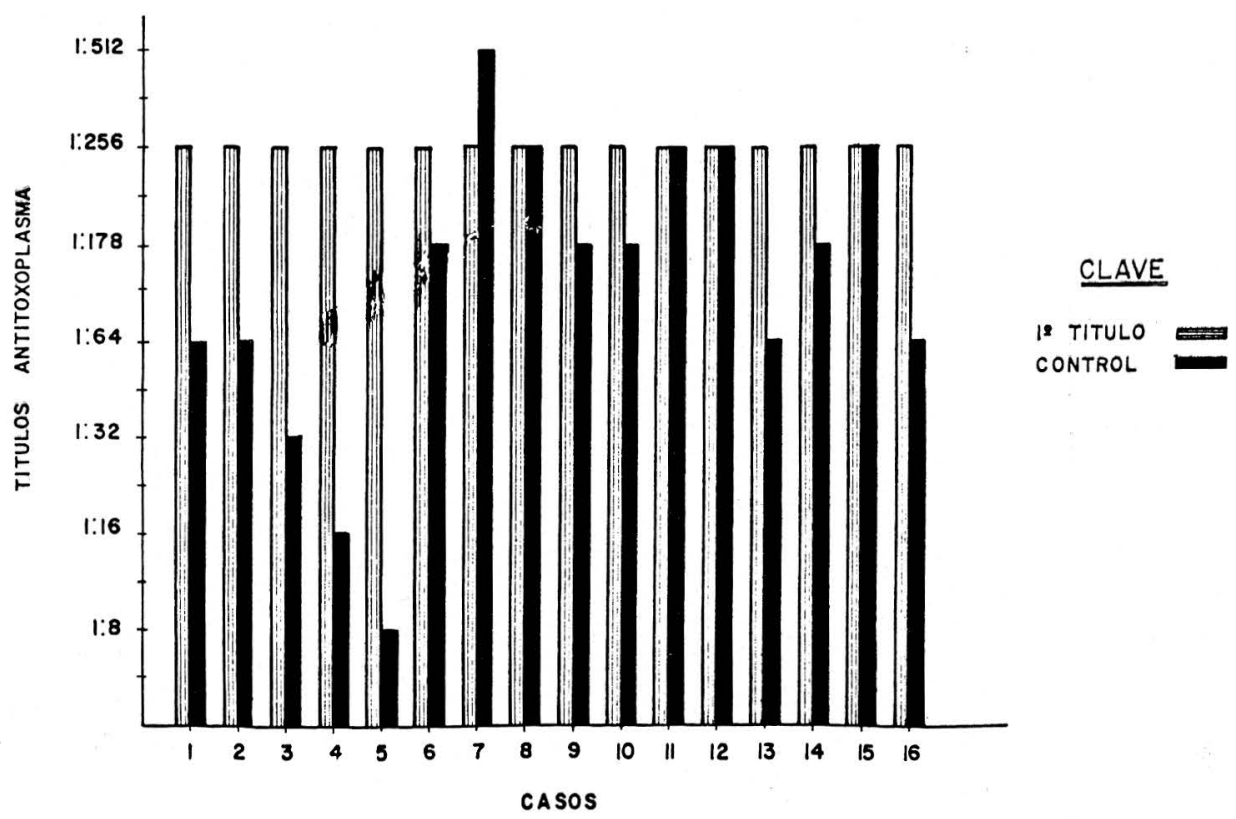

Cuadro No. 2

\section{RELACION DE ANTECEDENTES} EPIDEMIOLOGICOS EN TITULOS AC ANTITOXOPLASMA

\begin{tabular}{|lcc|cc|cc|}
\multicolumn{9}{c}{ MADRES } \\
\begin{tabular}{|lcc|ccc|cc|}
\hline & N2 & $\%$ & N2 & $\%$ & N2 & $\%$ \\
\hline $\begin{array}{l}\text { CONVIVENCIA } \\
\text { CON GATOS }\end{array}$ & 13 & 6.6 & 17 & 3.4 & 50 & 10 \\
\hline $\begin{array}{l}\text { INGESTION DE } \\
\text { CARNE SEMI- } \\
\text { CRUDA }\end{array}$ & 76 & 15.2 & 28 & 5.6 & 104 & 20.8 \\
\hline NINGUNO & 106 & 21.2 & 240 & 48 & 346 & 69.2 \\
\hline TOTAL & 215 & 43 & 285 & 57 & 500 & 100 \\
\hline
\end{tabular}
\end{tabular}

En los hijos de las 7 madres con títulos positivos para la enfermedad se encontraron valores de $\operatorname{lgM}$ por encima de 1:64 en 2 niños; en 3 éstos títulos fueron negativos, mientras los valores de IgG fueron superiores a 1:64 en 3 de los niños y en 2 fueron de 1:16. Al momento de analizar esta información, 2 niños aún no habían nacido, (Gráfica No. 7).

En el grupo de madres reactivas, el $6.6 \%$ informó convivencia con gatos, el $15.2 \%$ ingestión de carne semicruda $y$ el $21.2 \%$ no informó ninguno de estos antecedentes. 
En el grupo de las madres no reactivas, el $3.4 \%$ informó convivencia con gatos, el $5.6 \%$ ingestión de carne semicruda $y$ el $48.0 \%$ no informó ninguno de estos antecedentes.
En el total de madres estudiadas, el $10.0 \%$ informó sobre convivencia con gatos, el $20.8 \%$ sobre ingestión de carne semicruda, mientras el $69.2 \%$ no informó ninguno de estos antecedentes. (Cuadro No. 2).

\author{
Gráfico No. 6
}

\title{
SEGUIMIENTO DE TITULOS ANTITOXOPLASMA \\ + PARA ENFERMEDAD (MADRES)
}

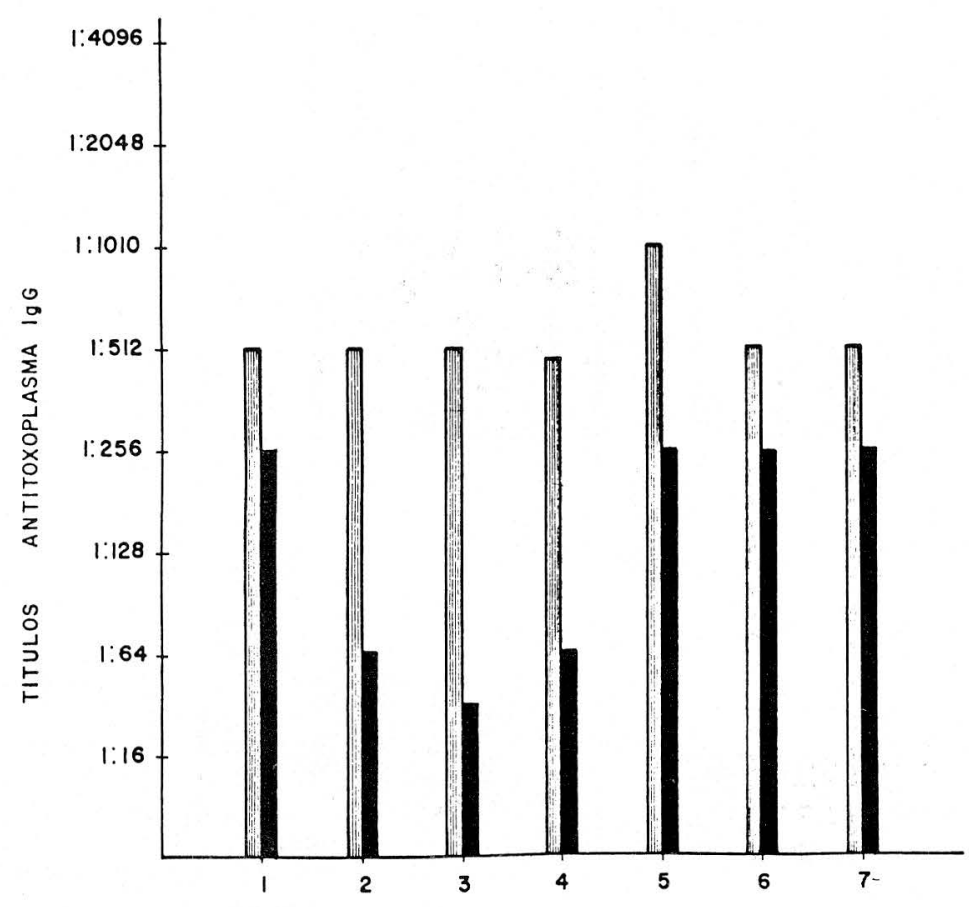

CLAVE

PRETTO

POSTTO

La edad gestacional en semanas lunares fue de 36 semanas en el caso No. 1 $y$ de 38 en el caso No. 2; ambos nacieron por parto espontáneo en presentación cefálica y ambos nacieron meconiados, el caso 1 Grado I y en el caso 2 con
Grado III. En el primero de los casos se encontró una placenta grande $y$ el segundo presentaba placenta pequeña con infartos. La clasificación de apgar fue a los 5 minutos de $8 / 10$ y de $7 / 10$ respectivamente (Cuadro No. 3 ). 
Gráfica No. 7

\section{TITULOS AC ANTITOXOPLASMA IgG - IgM EN LOS HIJOS DE LAS MADRES POSITIVAS PARA ENFERMEDAD}

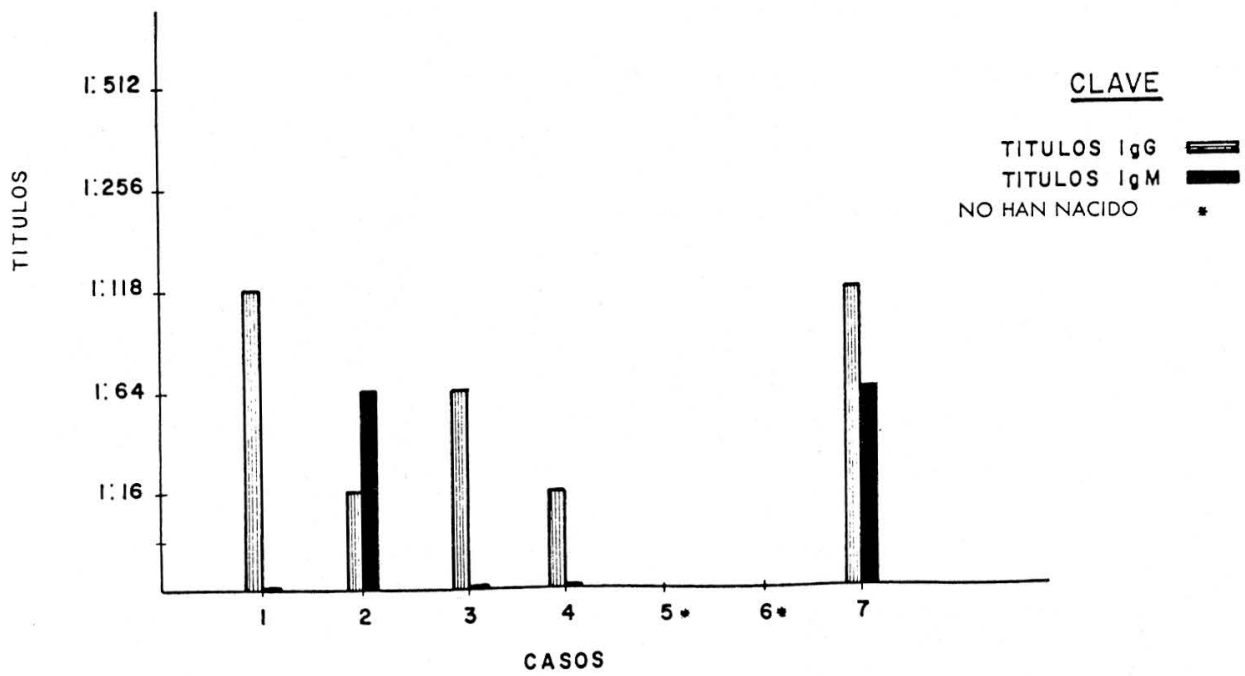

Cuadro No. 3

\section{CARACTERISTICAS DEL PARTO}

\section{CASO №1}

CASO №2

- E. Gestacional: $36 \mathrm{~S}$

- E. Gestacional: $38 \mathrm{~S}$

- Espontáneo cefálico

- Meconiado G I

- Espontáneo cefálico

- Meconiado G III

- Plancenta (macro) normal

- Placenta pequeña (300g Infortos

- Apgar 5': $8 / 10$

- Apgar 5: $7 / 10$

Caso No. 1

Los títulos de Anticuerpos Antitoxoplasma tomados de sangre del cordón fueron: IgM 1:4.006, IgG 1:126.

El paciente recibió tratamiento pero en el curso de éste murió por causas asociadas al severo retardo del crecimiento intrauterino.
CLINICA: CARACTERISTICAS SOMATOMETRICAS DE LOS 2 CASOS CON SOSPECHA DE TOXOPLASMOSIS

CASO № I

CASO № 2

- Sexo:M - Sexo:M

- P: $1.540 \mathrm{gr}$.

- P: $2200 \mathrm{gr}$. Talla 48

- PC: $29.5 \mathrm{~cm}(\mathrm{~N}: 33 \mathrm{~cm})$

- PC: $29 \mathrm{~cm}(\mathrm{~N}: 34.2 \mathrm{~cm})$

_PT: $28 \mathrm{~cm}(\mathrm{~N}: 30 \mathrm{~cm})$ - PT: $30 \mathrm{~cm}(\mathrm{~N}: 32 \mathrm{~cm})$

Con relación a los valores de referencia (37), desde el nacimiento y durante un lapso de 5 meses el perímetro cefálico se encontró francamente por debajo de los valores de referencia, sin embargo se aprecia una tendencia al incremento durante este tiempo desde un valor inicial de $29.0 \mathrm{~cm}$. hasta $34.5 \mathrm{~cm}$, (Gráfica No. 8). 
CLINICA: CARACTERISTICAS CLINICAS DE LOS 2 CASOS CON SOSPECHA DE TOXOPLASMOSIS

CASO №I

CASO № 2

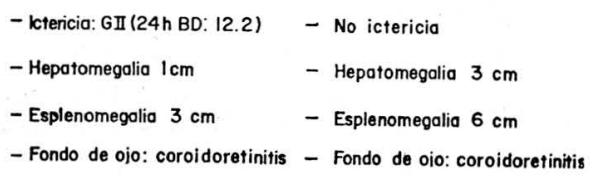

Gráfico No. 8

\section{RELACION DEL PC CON LA EDAD}

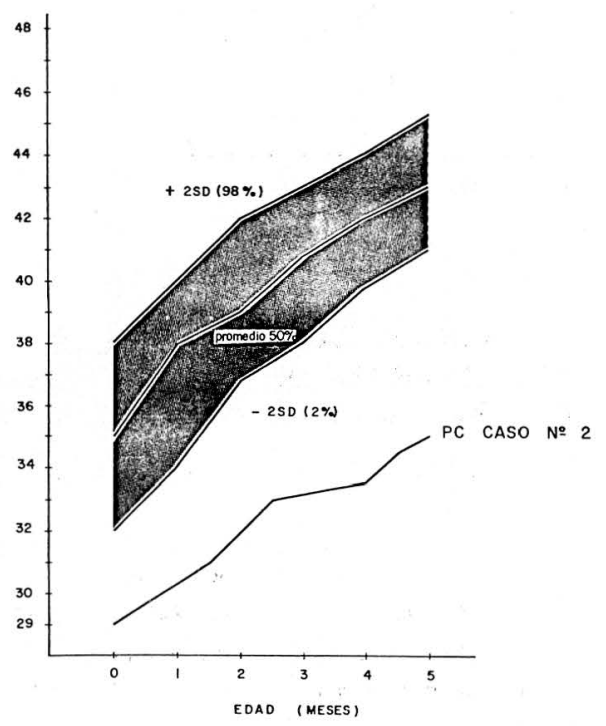

Caso No. 2

Los valores de hemoglobina se encontraron dentro de límites normales (37) durante un período de 5 meses subiendo en los días inmediatamente posteriores al tratamiento luego de los cuales se aplicaron transfusiones (con glóbulos rojos empaquetados $10 \mathrm{cc} / \mathrm{kg}$ ). (Gráfica No. 9).
Gráfica No. 9

\section{RELACION DE VALORES HB SEGUN LA EDAD}

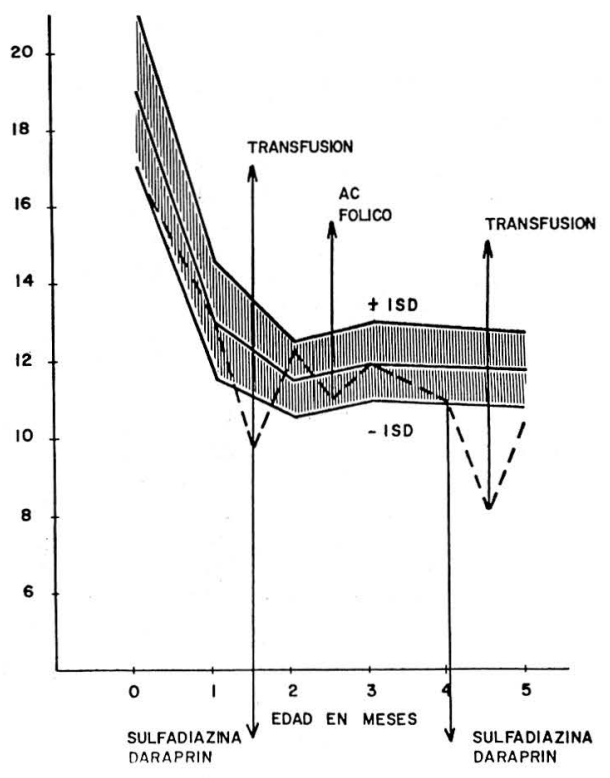

Caso No. 2

Los valores de IgG de su hijo descendieron hasta 1:256 a los 2 meses, estabilizándose durante 15 días y a partir de los 2 meses y medio ascendieron rapidamente hasta valores de 1:2.048 a los 4 meses y medio.

Los valores de $\lg G$ en la madre descendieron de 1:512 a 1:256 un mes después del parto y volvieron a ascender progresivamente a partir de esta fecha hasta valores de 1:4.096 a los 4 y medio meses después del parto.

Los valores de IgM del niño ascendieron desde 1:64 al nacer, hasta 1:128 a los 2 meses de nacido, a partir de los cuales descendieron hasta 1:68 a los 4 meses y medio de edad. (Gráfico No. 10). 
Gráfico No. 10

\section{VALORES DE AC ANTITOXOPLASMA DEL CASO No. 2}

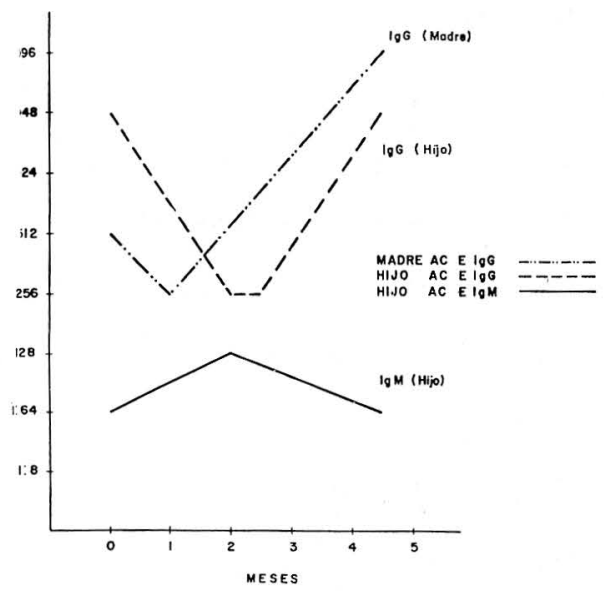

\section{Comentarios y Conclusiones}

Del análisis de los resultados anteriores se desprende que existe un problema severo de infestación por Toxoplasma Gondii en la población colombiana; a pesar de que la virulencia del parásito parece ser elevada en términos de comunidad, en todas las clases socio-económicas estudiadas, la patogenia aparente de la entidad podría interpretarse como relativamente baja. Sin embargo, desde el punto de vista eugenésico y dada la irreversibilidad de las lesiones fetales, debe determinarse la existencia de infestación por Toxoplasma Gondii en todas las embarazadas, especialmente en aquellas que tienen antecedentes de aborto. Existe una franca relación en estos aspectos con la infestación por el parásito, toda vez que los resultados muestran un mayor número de abortos en las madres cuyo título de Anticuerpos Antitoxoplasma fue reactivo de aquellas en las cuales no lo fue.
Igualmente, desde el punto de vista epidemiológico parece jugar un papel importante la convivencia con gatos y la ingestión de carnes con deficiente cocción. El hecho de que la infestación curse sin signología clínica definida, obliga definitivamente a disponer de determinaciones de laboratorio inmunológico con fines de diagnóstico, tratamiento $y$ prevención de lesiones en el feto.

El tratamiento utilizado demostró ser relativamente efectivo; sin embargo, queda la posibilidad de reinfecciones tanto durante la aplicación de éste como a su suspensión.

En los hijos de las madres estudiadas se demostró la posibilidad de transmisión de la parasitosis al feto durante la vida iritrauterina, de acuerdo a los valores de IgM encontrados en 2 de los niños, así como la importancia del tratamiento oportuno durante la gestación con fines de protección del feto. En la misma forma se evidenció la transferencia de anticuerpos de madre a hijo, para la enfermedad.

En los dos casos de referencia estudiados se demostraron las alteraciones placentarias probablemente debidas a la infestación, tanto como grados variados de sufrimiento fetal con retardo franco del crecimiento intrauterino y alteraciones viscerales $y$ de fondo de ojo, las cuales por lo menos en el caso del perímetro cefálico persistieron en valores subnormales en la vida extrauterina a pesar del tratamiento.

Se comprobó enfermedad activa en los dos casos de referencia por valores de IgM positivos y en el caso No. 2 se aprecia el descenso de dichos valores posterior al tratamiento, ésto corrobora la efectividad de la terapéutica y su importancia en relación a los mínimos efectos colaterales encontrados. El ascenso sostenido de los títulos de IgG de madre e hijo (caso No. 2), confirma la presencia de infestación toxoplásmica. Se eviden- 
ció que el tratamiento presenta efectos secundarios de severidad discreta y de corrección relativamente fácil, tales como la caída de la hemoglobina.

Vale tener en cuenta que las condiciones ecológicas donde se adquirió la enfermedad no fueron modificadas en el estudio.

Los resultados obtenidos en cuanto a infección toxoplásmica ameritan la realización de estudios prospectivos de tipo analítico-experimental, en grupos de población representativos, con el fin de establecer las características epidemiológicas de la infestación por Toxoplasma Gondii, la cual, de acuerdo a lo encontrado, es sugerente de un comportamiento endémico especialmente en la población de mujeres en edades reproductivas, tanto como en la de animales domésticos.

En la misma forma desde el punto de vista eugenésico vale la pena definir la participación del toxoplasma como causa determinante asociada de la presentación de abortos espontáneos, lo cual podría estar relacionado con el escaso número de lesiones en el neonato, en el sentido de que la mayoría de las lesiones en los períodos tempranos de la gestación determinarian la escasa viabilidad de los productos de gestación por lesiones de gran magnitud en periodos críticos de la organogénesis.

\section{Resumen}

Se presentan los resultados de un estudio descriptivo sobre la prevalencia de infestación de Toxoplasma Gondii, en un grupo de madres y sus productos de gestación, asistente a la consulta externa de Ginecología y Obstetricia del Hospital Infantil Lorencita Villegas de Santos de Bogotá, Colombia. Se encontró una importante prevalencia de infestación parasitaria en madres e hijos, determinándose antecedentes importantes de abortos, en las madres parasitadas, mientras en los productos de gestación las lesiones atribuibles a Toxoplasma Gondii fueron mínimas.

El estudio sugiere la existencia de un grave problema de salud pública, de alta virulencia, cuya patogenicidad puede estar enmascarada por el mínimo número de lesiones en los productos de gestación viables, si no se tiene en cuenta el mayor número de abortos que se informaron en las madres parasitadas.

Igualmente se destaca la importancia de la ingestión de carne deficientemente cocida y la convivencia con mamíferos de sangre caliente (gatos), como vectores potenciales del parásito.

\section{PREVALENCE OF TOXOPLASMA GONDII IN A GROUP OF MOTHERS AND THEIR PRODUCTS OF CONCEPTION}

\section{Summary}

This article presents the outcomes of a descriptive study on the prevalence of infestation of Toxoplasma Gondii in a group of mothers and their products of conception, seen at the Gyn out-patient service at Lorencita Villegas de Santos Hospital in Bogotá, Colombia. The study determined an important prevalence of parasitic infestation in mothers and offspring, a high incidence of abortions in infested mothers, and a low incidence of damage related to Toxoplasma Gondii in the foetuses.

The study suggests the existence of a highly harmful public health problem whose pathogenic effects might be masked by the realtively low incidence of 
symptoms in viable foetuses, and not taking into consideration the highernumber of abortions in infested mothers as mentioned above.

\section{Bibliografía}

1. LAUGIER, GIRARD PAUL, GIRARD HENRY. Problems poses por la diagnostic serolguique de la toxoplasmose chez $L^{\prime}$ enfant. Arch. Franc de Pediat. Tomo 22 No. 10: 1.201, 1965.

2. ARBELAEZ, L., MARTINEZ, H., Toxoplasmosis congénita. Presentación y discusión de un caso clínico. Antioquia Med. Vol. 21 No. 4: 249-272, 1971.

3. MARTIN SANZ. Toxoplasmosis, Datos de interés clínico. Bol. Fund. Jiménez Díaz. Vol. III No. 12: 675, 1971.

4. ROCA GARCIA, M., CAMACHO GAMBA, J., ESGUERRA GOMEZ, G. Un caso de toxoplasmosis congénita. Rev. Col. de Ped. y Puer, 10, 5: 238-276, 1951.

5. TORRES, C., Un caso de Toxoplasmosis. Rev, Col, de Ped, y Puer. 14: 407-411 1955.

6. RODRIGUEZ GOMEZ, A: Toxoplasmosis en Colombia. Rev. Col. de Ped. y Puer. XV, 5: 308, 1956.

7. MUÑOZ RIVAS, G., Toxoplasmosis congénita en Colombia. Rev. Inst. Salubr. y Enfer. Trop. Vol. 19: 531-355, 1959.

8. LAVERDE, A, y ARCINIEGAS, J. Segundo caso Colombiano de Toxoplasmosis Congénita. Ann, Soc. Biol. Bogotá, 5: 237-243, 1953.

9. RESTREPO, M., y ROBLEDO, M. Toxoplasmosis Congénita. Estudio clínicopatológico de ocho casos. Antioquia Med. 15: 320, 1965.
The article also stresses the importance of undercooked meats and the proximity of hot-blooded mammmals (cats) as potential vectors of the parasite.

10. PiESTREPO, M., JARAMILLO VICENTE, KURSER, A. Toxoplasmosis congénita. Presentación de un caso diagnosticado en vida con aislamiento del parásito. Antioquia, Med. Vol. 20: 309314, 1970.

11. BARRIOS, H. Un caso de toxoplasmosis congénita. Rev. Med. Cir. Barranquilla, 20: $18-32,1953$.

12. NICOLLE, C., MANCEAUX, L. Sur une infection a corps de Leishman due gondi. Compt. Rend. Acad. Sci. 147: 763-766, 1908.

13. BOTERO DAVID, LONDOÑO, O., RESTREPO M. Estudio sobre Toxoplasmosis Humana en Colombia. Antioquia Med. 15: 325, 1965.

14. REMINGTON, J.S., DESMONTS, G., Congenital Toxoplasmosis: variability in the $\mathrm{lg} . \mathrm{M}$ fluorescent antibody response and some pitfalls in diagnosis. J. Pediatri. 83: $27-30,1973$.

15. KIMBALL, A.C., KEAN, S.H., FUCUS, F. Toxoplasmosis risk variations in New York City Obstetric Patients. Am. Jour, Obstet. Gynecology Vol. 119, 208-214, 1974.

16. DESMONTS, G., COUVREUR, J. Congenital Toxoplasmosis; a prospective study of 378 pregnancies. New Engl. Jour, of Med. 290: 1.110-1.116, 1974.

17. CRISCELLI CLAUDE, DESMONTS, G. FROMMEL DOMINIQUE. Congenital To. xoplasmosis. Fetal Synthesis of oligocional Inmunoglobin Gin Intrauterine infection. Journal of Pediatr. 83: Nol: 20-26, 1973. 
18. DESMONTS, G., GERBEAU, J., LELONG, M. Les modes de propagation de la toxoplasmose humaine. Archiv. Francaises de Ped. 18: 1.026-1.032, 1961.

19. TERRIS, J., MUSSET, R. Enquete sur la toxoplasmose chez 1.858 femmes enceintes. Gyn. Obst. (París) 68 No. 1: 25-34, 1966.

20 GARRIS, J., DESMONTS, G., Colloque sur la toxoplasmose de la femme enceinte et la prevention de la toxoplasmose congenitale. Lyon medical 12-10: $2 €$ $-28,1969$.

21. RENARD, G., LEBVISSON, D. La toxoplasmose. Le pint de vue de L'ophtal* mologiste. Le concours Medical. 25, 3, 1978.

22. CHABBERT, Y., Etudes in vitro sur la spiramicine activite, resitance, antibiogramm concentrations humorales. Annales de L'institut Pasteur, Vol. 89, No. 4: 434-446, 1955.

23. STRAY BABILL, PEDERSON. Infants Potentially at risk for congenital toxoplasmosis. A. prospective study. Am. J. Dis. Child. Vol. 134: 638, 1980.

24. ANGEL, G., Toxoplasmosis en el embarazo y sus manifestaciones en el recién nacido. Laboratorio médico y Ginecológico. Boletín de laboratorio No. 99, 1981.

25. GIRARD PAUL, GIRARD HENRY, LANGIER. Diagnostic et traiment de la toxoplasmose humaine congenitale ou acquise de I'enfant. La revuede Medecine clinique de Pediatría et Puericulture 11, 127, 1963.

26. MARTIN SANZ. Toxoplasmosis. Datos de interés clínico. Bol. Fund. Jiménez, Día. Vol. III No. 12, 675, 1971.
27. REMINGTON, J., DESMONTS, Toxoplasmosis, Infections Diseases of the fetus and $\mathrm{N}$ infant, Philadelphia, N B Saunders Co. 191, 1976.

28. STAGNO SERGIO; Congenital toxoplasmosis Marginal Camment. Am J. Dischild: Vol. 134, 635, 1980.

29. SABIN, A.B. Toxoplasma encephalitis in children, JAMA 116: 801-807, 1941.

30. DESMONTS, G. et al Arch. Franc. Estud. Clin. Biol. 22: 1.183-1.200, 1965.

31. SWARTZBERG, J. y REMINGTON, J.S. Transmission of toxoplasma. Am. J. Dis. Child 129: 777-779, 1975.

32. EICHENWALD, J. Experimental toxoplasmosis 1, Transmission of infection in utero and through the milk of lactating female mice. Am. J. Dis Child 76: 307$315,1948$.

33. APT. W. "Transmisión Congénita". Estado actual del problema. I. Introducción y Generalidades. Bol. Chile parasit. 19: 31-37, 1964.

34. REMINGTON. J.S. Toxoplasmosis, Obstetric and perinatal infections 27-24, 1973.

35. APT. W. Transmisión Congénita de protozoos parásitos. Boletín de la oficina Sanitaria Panamericana 517-543, 1972.

36. ALFORD, C.A. Jr., STAGNO, S. y Reynolds, D.W. Congenital Toxoplasmosis; clinical, laboratory, and therapeutic considerations, with special reference to subclinical disease. Bull. N.Y. Acad. Med. 50: 160-181, 1974.

37. MARSHALL, H. Klaus. Fanaroff Avroy Clasificación del recién nacido de bajo peso. Asistencia del recién nacido de alto riesgo. Editorial Médico Panamerica. no. Viamonte. 2.164 Buenos Aires. 80, 1981 